\title{
Expression and gene regulatory network of SNHG1 in hepatocellular carcinoma
}

\author{
Chaoran Zheng $^{1 *}$ and Shicheng $\mathrm{Yu}^{1,2,3^{*}}$ (D)
}

\begin{abstract}
Background: Small nucleolar RNA host gene 1 (SNHG1), a long noncoding RNA (IncRNA), is a transcript that negatively regulates tumour suppressor genes, such as p53. Abnormal SNHG1 expression is associated with cell proliferation and cancer. We used sequencing data downloaded from Genomic Data Commons to analyse the expression and interaction networks of SNHG1 in hepatocellular carcinoma (HCC).

Methods: Expression was examined using the limma package of $\mathrm{R}$ and verified by Gene Expression Profiling Interactive Analysis. We also obtained miRNA expression data from StarBase to determine the IncRNA-miRNA-mRNA-related RNA regulatory network in HCC. Kaplan-Meier (KM) analysis was performed using the survival package of R. Gene Ontology annotation of genes was carried out using Metascape.

Results: We found that SNHG1 was overexpressed and often amplified in HCC patients. In addition, SNHG1 upregulation was associated with the promotion of several primary biological functions, including cell proliferation, transcription and protein binding. Moreover, we found similar trends of small nucleolar RNA host gene 1 (SNHG1), E2F8 (E2F transcription factor 8), FANCE (FA complementation group E) and LMNB2 (encodes lamin B2) expression. In the SNHG1associated network, high expression levels of SNHG1 (log-rank P value $=0.0643$ ), E2F8 (log-rank P value $=0.000048$ ), FANCE (log-rank $P$ value $=0.00125)$ and LMNB2 (log-rank $P$ value $=0.0392$ ) were significantly associated with poor survival. Single-cell analysis showed that E2F8 may play an important role in tumorigenesis or cancer development.
\end{abstract}

Conclusions: Our results highlight the benefit of utilizing multiple datasets to understand the functional potential regulatory networks of SNHG1 and the role of SNHG1 in tumours.

Keywords: $L I H C$, HCC, TCGA, ceRNA, SNHG1, Survival

\section{Background}

Liver cancer is the fourth leading cause of death from cancer in the world [1] and is one of the most fatal and algetic tumours. Hepatocellular carcinoma (HCC) is the most common histological type of primary liver cancer, occurring in $75-85 \%$ of patients with primary liver cancer, and Africa and Asia have the highest incidences [1,

\footnotetext{
*Correspondence: zhengchaoran@whu.edu.cn; yu_shicheng@gibh.ac.cn; shyu@whu.edu.cn

${ }^{1}$ Key Laboratory of Combinatorial Biosynthesis and Drug Discovery, School of Pharmaceutical Sciences, Wuhan University, Wuhan 430071 China

Full list of author information is available at the end of the article
}

2]. Recent research shows that HCC patients tend to have a family history of HCC and that the pathological mechanisms are complex [3, 4]. Overall, medical practice has changed rapidly in the last few years with the aid of rapidly expanding genome sequencing techniques [5], and precision medicine is gradually becoming an emerging field in the treatment and prevention of diseases. Although medical researchers have overcome the challenges of assessing large amounts of data, the application of genetic testing in cancer treatment is progressing slowly. A primary reason for this slow progress is that large amounts of accumulated patient data are independent and discrete. Thus, researchers need to determine

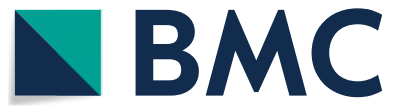

(c) The Author(s) 2021. This article is licensed under a Creative Commons Attribution 4.0 International License, which permits use, sharing, adaptation, distribution and reproduction in any medium or format, as long as you give appropriate credit to the original author(s) and the source, provide a link to the Creative Commons licence, and indicate if changes were made. The images or other third party material in this article are included in the article's Creative Commons licence, unless indicated otherwise in a credit line to the material. If material is not included in the article's Creative Commons licence and your intended use is not permitted by statutory regulation or exceeds the permitted use, you will need to obtain permission directly from the copyright holder. To view a copy of this licence, visit http://creativecommons.org/licenses/by/4.0/. The Creative Commons Public Domain Dedication waiver (http://creativecommons.org/publicdomain/zero/1.0/) applies to the data made available in this article, unless otherwise stated in a credit line to the data. 
relationships between diverse data and their correlations with disease.

Noncoding RNAs (ncRNAs) are a class of RNA molecules that do not participate in protein coding but do regulate gene expression, contributing to many physiological and pathological processes, including tumours [6]. Long noncoding RNAs (lncRNAs) are an important subcategory of ncRNAs with lengths longer than 200 nucleotides $[7,8]$. Recent studies show that lncRNAs participate in various biological processes, such as cell differentiation [9], signal transduction [10] and posttranscriptional regulation [11], that are conducive to the progression of tumours and many other diseases [12]. Increasing evidence suggests that lncRNAs can function alone or in cooperation with microRNAs (miRNAs). Typically, lncRNAs act as ceRNAs to regulate messenger RNAs (mRNAs). This mode is known as the "IncRNA-miRNAmRNA network". This regulation has been reported in some tumour-related studies, but traditional research methods are insufficient for analysing multi-omics data $[13,14]$. Nevertheless, ceRNA network analysis can shed light on ncRNA expression alterations in cancers and uncover relationships between altered RNAs.

To reveal the roles of the lncRNA-miRNA-mRNA network in human HCC, we sought to identify ceRNA networks by identifying similar regulatory networks from an extensive collection of data from Genomic Data Commons (GDC) using a stringent bioinformatics pipeline. The identified ceRNA networks may help in identifying molecular subtypes or new biological markers to classify HCC patients. Moreover, by examining differences in patient survival based on the expression of each RNA subtype, our ceRNA networks offer potential clinical utility for personalized treatments and therapeutic targets.

\section{Methods}

\section{Data downloading and organization}

The gdc-client method was used to download RNAseq, miRNA and clinical data from the GDC database via TCGA-Assembler (http://www.compgenome.org/ TCGA-Assembler/) of R (3.6.2) [15]. A total of 424 RNA-seq, 425 miRNA and 377 clinical data points were included in the study. Duplicates were filtered out before analysis of the metadata download from the GDC database. We also removed samples that were not from primary tumours or normal solid tissues. The TMM method implemented in edgeR of $R$ (3.6.2) [16] was used to normalize RNA-seq and miRNA raw count data, after which we removed genes with a logcpm of expression below 1 in more than half of the samples. We used the limma [17] package in R (3.6.2) (https://www.r-project.org/) to screen genes and miRNAs showing significant differential expression between tumour and normal tissues.
Significant differentially expressed genes (DEGs) (based on $\mathrm{P}<0.01$ and fold change $(\mathrm{FC})>2$ ) were identified in the RNA-seq and miRNA expression profiles, and volcano plots and bar plots were constructed in $\mathrm{R}$ to visualize these DEGs. Expression analysis of different sample types (tumour vs. normal tissues) was performed with GEPIA (http://gepia.cancer-pku.cn/) [18]. Next, we assessed the levels of protein expression for these genes based on immunohistochemistry data obtained from the Human Protein Atlas database (https://www.proteinatl as.org/) [19-21].

\section{ceRNA network analysis}

Competing lncRNA-mRNA pairs were identified using the gdcCEAnalysis function of GDCRNATools [15]. The precise identification of IncRNA-miRNA-mRNA interactions was based on three lines of evidence. First, the hypergeometry of shared miRNAs was evaluated to reduce the number of lncRNA-mRNA pairs; hypergeometric analysis was performed to determine whether a lncRNA-mRNA pair shares many miRNAs. Second, we excluded all IncRNA and mRNA pairs that exhibited variable correlations in expression. Finally, the lncRNAmRNA pairs retained were required to have regulation similar to that of all shared miRNAs. After these steps, several lncRNA-miRNA-mRNA interactions were identified, and functional analysis was then carried out. To identify the number of miRNAs related to lncRNAs and mRNAs, miRNA-mRNA and miRNA-lncRNA interactions were obtained from StarBase v2.0 [22]. The network was visualized using Cytoscape (3.4.0) (https://cytoscape. org) $[23,24]$.

\section{Univariate survival analysis}

GdcSurvival Analysis in GDCRNATools was employed to analyse the association with overall survival for each gene [15]. First, we divided the patients into high- and low-expression groups based on the median expression level. Then, Kaplan-Meier (KM) analysis was performed using the survival package, and the results were examined in univariate survival analysis. The final results are presented as the hazard ratio (HR) and significance (logrank $P$ value).

\section{Functional analysis}

Gene Ontology (GO) annotation for the genes was carried out using the Metascape database (http://metas cape.org/gp/index.html\#/main/step1) [25]. Then, Online Mendelian Inheritance in Man (OMIM) (https://www. omim.org/) was applied to analyse associations between diseases and the genes in the lncRNA-miRNA-mRNA network. 


\section{Single-cell analysis}

To assess gene expression in different cell types, we obtained single-cell sequencing data from a previous report [26]. Gene expression was normalized by log2 (filteredexpr +1 ) as mentioned in [26]. Cell clusters were generated with seurat software [27], and the cell clustering information was retrieved from the same report [26]. The expression of these genes in endothelial cells and macrophages was evaluated, and the results of gene expression in different cell clusters are illustrated in a violin plot.

\section{Results}

\section{RNA expression in HCC}

Using the limma package, we identified 807 upregulated DEGs, 1590 downregulated DEGs, 59 upregulated differentially expressed miRNAs (DEMs), and 71 downregulated DEMs (Fig. 1). In total, we identified 2397 DEGs and 130 DEMs.

\section{Biological interaction networks in HCC}

We next sought to determine the biological interaction networks in $\mathrm{HCC}$ by using internally incorporated miRNA-mRNA and miRNA-lncRNA interactions, and we identified seven mRNA-miRNA-lncRNA networks (Fig. 2). All the ceRNA networks share similar regulation of all miRNAs interacting with the lncRNA-mRNA pairs. For example, the neighbouring genes of MAGI2AS3, namely, ADAMTSL3, AUTS2, BHLHE40, DUSP6, ERRFI1, GADD45A, GNPNAT1, HIVEP1, MYLK,
MYO10, PNRC1, RIPOR2, SMAD6, SPRY2, and SYBU, share the same miRNAs. Additionally, two miRNAs (hsa-miR-374a-5p and hsa-miR-374b-5p) mediated the MAGI2-AS3-mRNA network.

\section{Univariate survival analysis of coexpressed genes in HCC}

Abnormal expression of many genes affects the duration of human survival. By constructing a ceRNA network, we identified hub genes with which miRNAs might interact. In addition, we established survival-related networks. Then, we focused on SNHG1 and related genes that predict the same direction of correlation with prognosis.

We evaluated SNHG1 transcription levels in HCC studies from GDC. Data in the GDC database revealed significantly higher mRNA expression of SNHG1 in HCC samples than in normal solid tissue samples (log-rank $\mathrm{P}$ value $=6.43 \mathrm{E}-18)$ (Table 1$)$, with a fold difference above 2 . The results of the expression analysis for SNHG1, $L M N B 2, F A N C E$, and E2F8 were verified by GEPIA (Fig. 3). We also verified the low protein levels of these four genes in normal samples using the Human Protein Atlas database (Fig. 4). Four miRNAs (hsa-miR-421, hsamiR-377-3p, hsa-miR-330-5p, and hsa-miR-326) mediated the SNHG1 network. Thus, SNHG1 expression may serve as a prognostic indicator in HCC.

To better understand the functions of the coexpressed genes (E2F8, FANCE and LMNB2) correlated with SNHG1, we compared survival time among patients with different levels of gene expression and found that high expression of SNHG1 was associated with decreased
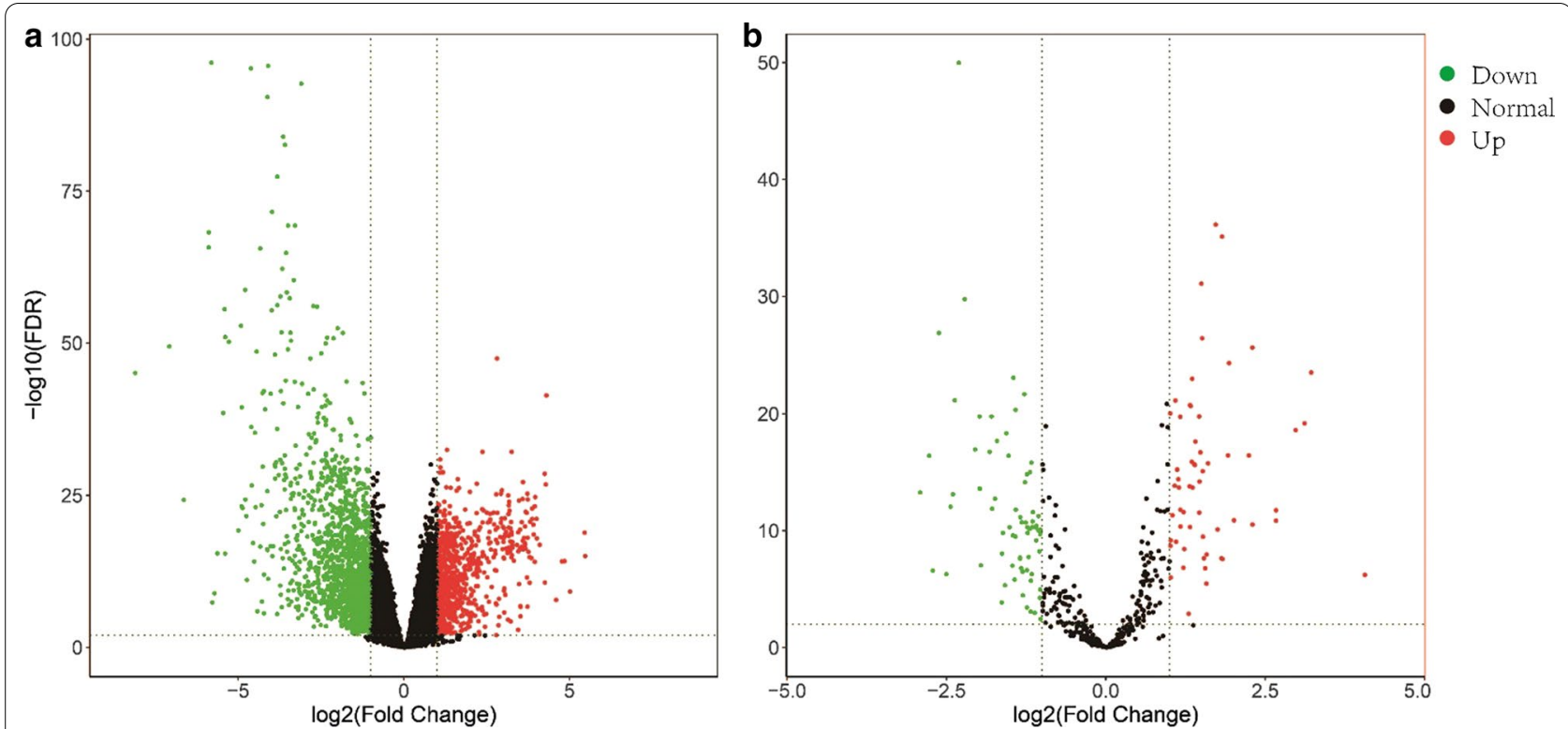

Fig. 1 Genes differentially expressed in HCC. A volcano plot was constructed in R with the expression matrix of DEGs (a) and DEMs (b), showing $\log 10$ (FDR) versus $\log 2$ (fold change) 


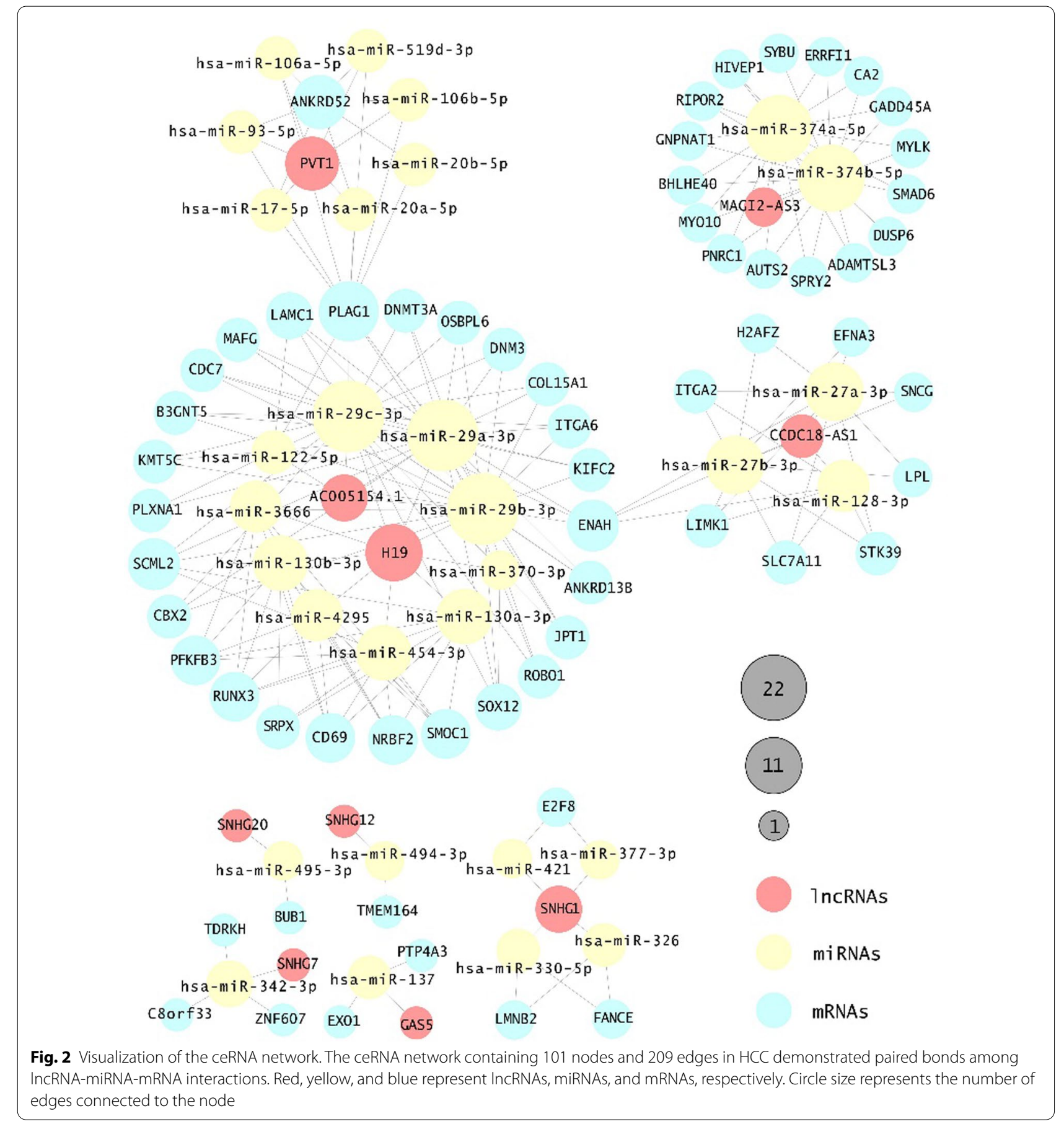

Table 1 Biological functions of the genes in the SNHG1-related network

\begin{tabular}{llllll}
\hline Gene symbol & Description & OMIM & Type & LogFC & P value \\
\hline SNHG1 & Small nucleolar RNA host gene 1 & 603222 & Long non coding & 1.495397836 & $6.43 \mathrm{E}-18$ \\
LMNB2 & E2F transcription factor 8 & 612047 & Protein coding & 1.115366226 & $5.95 \mathrm{E}-13$ \\
FANCE & FA complementation group E & 613976 & Protein coding & 1.459672439 & $9.12 \mathrm{E}-14$ \\
E2F8 & Lamin B2 & 150341 & Protein coding & 3.729047657 & $8.89 \mathrm{E}-27$ \\
\hline
\end{tabular}

The table lists 5 genes in the SNHG1-related network and the following information: Gene symbol, Description, OMIM, Type, LogFC (trimmed binary logarithm of average expression level fold change), and $\mathrm{P}$ value 


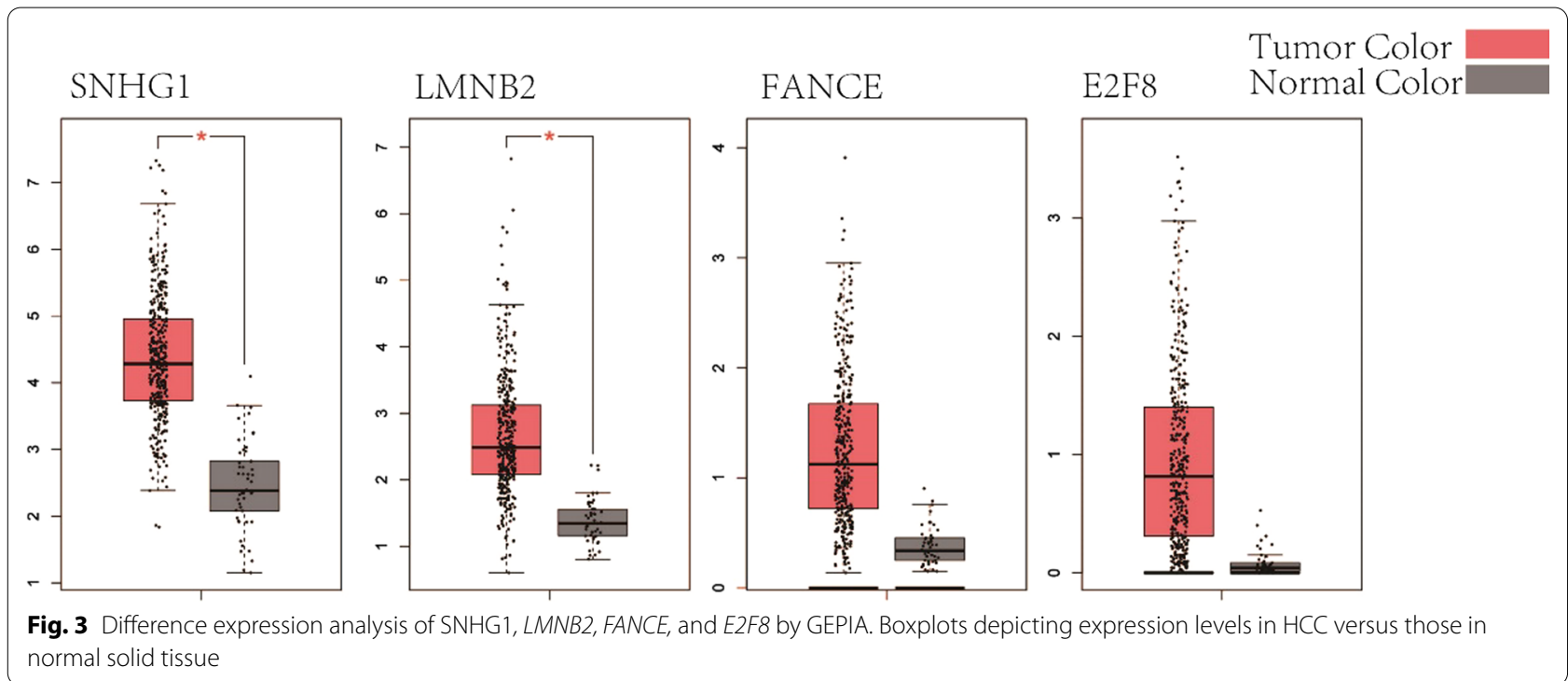

survival (log-rank $\mathrm{P}$ value $=0.0643$ ) (Fig. 5). Additionally, in the SNHG1-associated network, high expression levels of E2F8 (log-rank $\mathrm{P}$ value $=0.000048)$, FANCE (log-rank $\mathrm{P}$ value $=0.00125)$ and LMNB2 (log-rank $\mathrm{P}$ value $=0.0392$ ) correlated significantly with poor survival (Fig. 5); these genes affect transcription activity, DNA repair, and protein binding, respectively. Overall, we detected interaction effects of SNHG1 and the other three genes on survival.

\section{Functional analysis of the coexpressed genes correlated with SNHG 1 in HCC}

To examine the roles that the 3 coexpressed genes we identified play in the cell, we annotated all three genes and SNHG1 using the Custom Analysis function of Metascape (http://metascape.org/) (Table 1). All of these genes are involved in primary life processes, such as transcription activity, DNA repair, and protein binding. Two of these genes encode plasma proteins, and E2F8 encodes a transcription factor with a winged helix-turn-helix DNA-binding domain.

\section{SNHG1-related mRNA expression in endothelial and macrophage populations}

We analysed the expression of the three coexpressed genes described above in endothelial cells (ECs) and macrophages, which were significantly different based on single-cell data [26] (Figs. 6 and 7). LMNB2 was found to be uniformly expressed in the 11 EC clusters. Specifically, E2F8 was expressed at low levels in clusters $0,1,4$, and 8, corresponding to normal cells [26]. At the same time, FANCE was expressed at low levels in clusters 8 (adjacent normal), 9 (core tumour), and 10 (peripheral tumour), indicating no difference between tumour and normal cells. Mononuclear phagocyte-based subclustering analysis also suggested low levels of E2F8 expression in peripheral tumours and adjacent normal tissues. However, $L M N B 2$ and FANCE were uniformly expressed in the 9 macrophage clusters. These results suggest that several clusters of E2F8-deficient endothelial cells and macrophages may be tuned to regulate cancer processes in HCC.

\section{Discussion}

Using RNA-seq, miRNA and clinical data downloaded from the GDC database, we identified 807 and 1590 upregulated and downregulated DEGs and 59 and 71 upregulated and downregulated DEMs, respectively. A total of 2397 DEGs and 130 DEMs were found, and by constructing a ceRNA network, we detected hub genes with which miRNAs may interact. We observed 7 lncRNA-miRNA-mRNA interactions and performed survival analysis on all genes in the networks.

We analysed data for 377 patients from the GDC database and found that high SNHG1 expression indicated poor survival; high E2F8, FANCE and LMNB2 levels were also associated with poor patient survival. A previous study revealed a strong negative correlation between SNHG1 expression and survival time in patients with high-risk neuroblastoma (NB) [28]. In addition, downregulation of E2f8 resulted in a series of embryonic defects affecting vasculature and cell survival [29]. FANCE is reported to be a vital factor influencing the poor survival of FANCE-deficient cells among breast cancer cell lines [30], and upregulation of LMNB2 is associated with significantly poorer prognosis in lung 


\section{SNHG1}

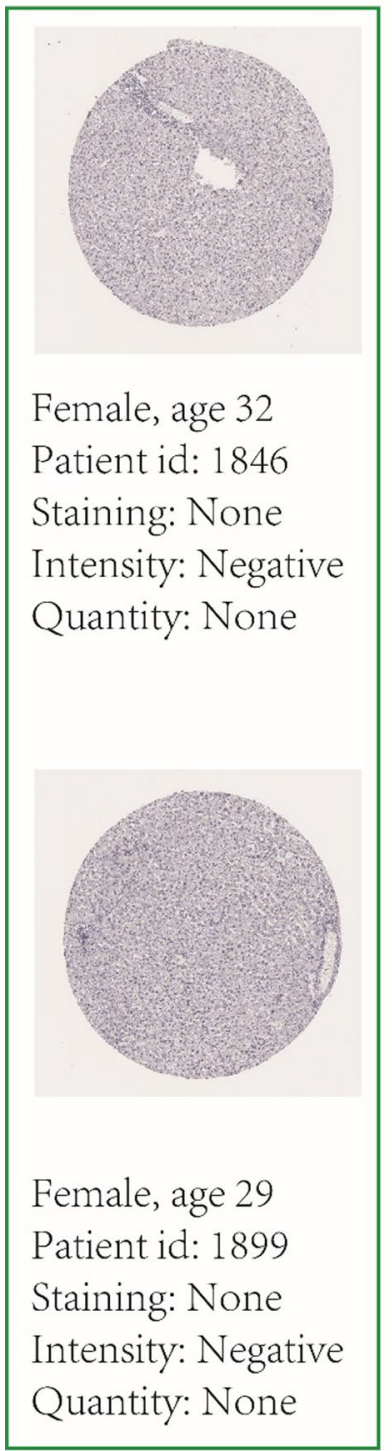

\section{LMNB2}

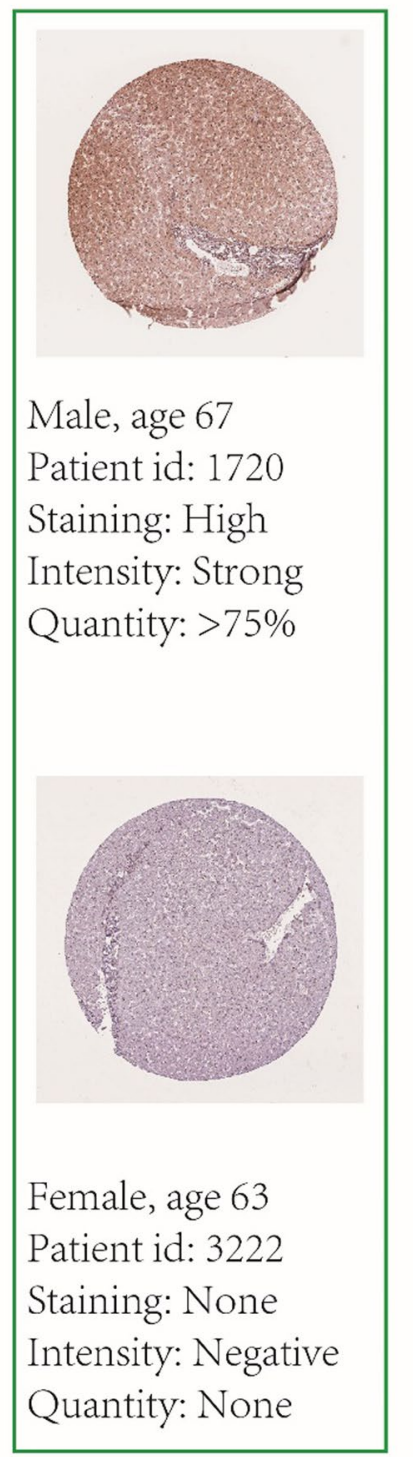

FANCE

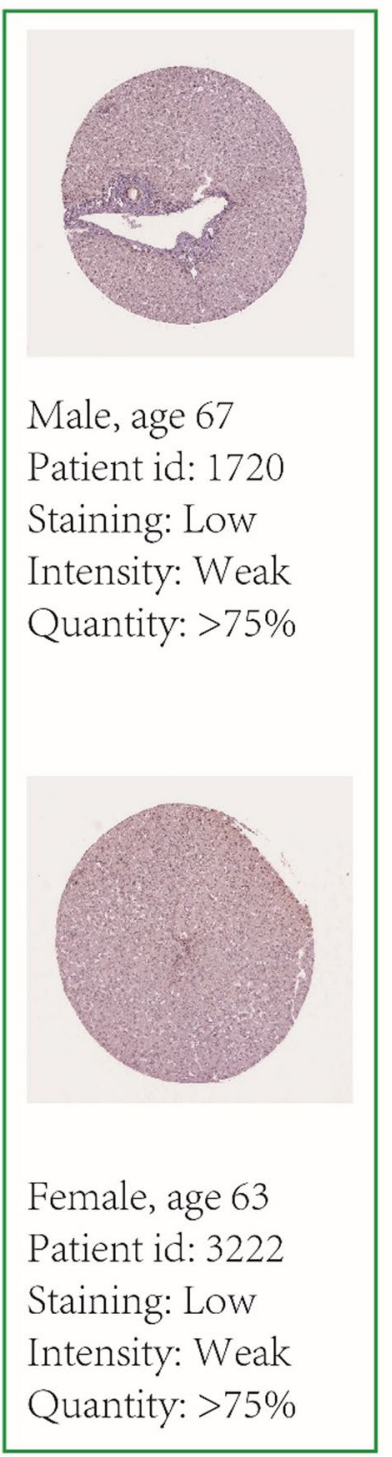

\section{E2F8}

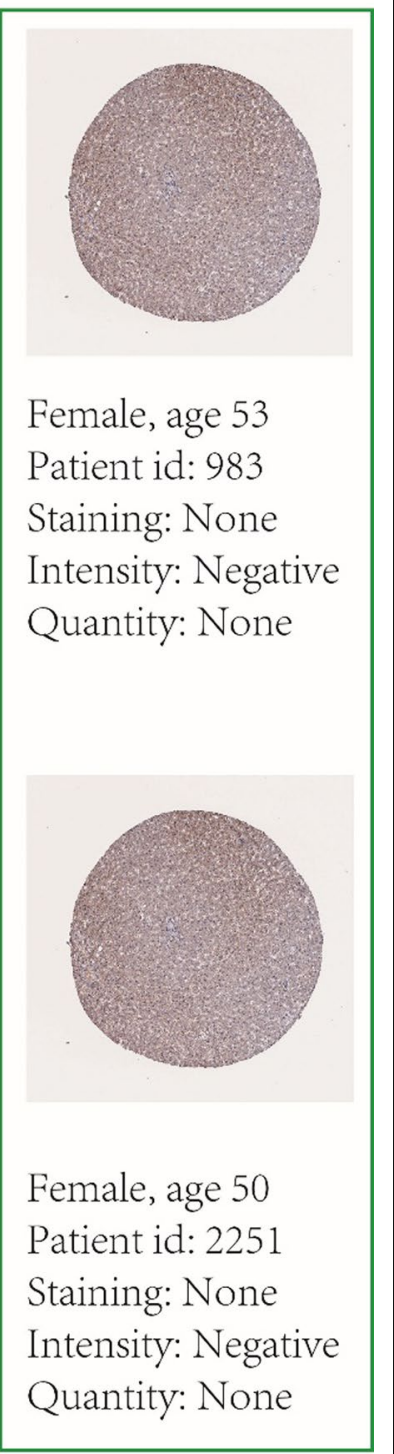

Fig. 4 Translational levels of SNHG1, LMNB2, FANCE, and E2F8 in Normal tissue. The immunohistochemistry result was obtained from Human Protein Atlas database. Number of tumour is 369; and number of normal is 50

adenocarcinoma patients [31]. Therefore, SNHG1 may affect survival through E2F8, FANCE and LMNB2. Further experiments are needed to examine interactions in this SNHG1-related network.

CeRNAs are RNA molecules that affect mRNA expression by competing for miRNAs in the same interaction network. Disruption of interactive networks can cause disease, including various cancers [32]. It is widely recognized that SNHG1 plays roles in cell proliferation and tumours. The patients showed poor prognosis when SNHG1 was upregulated, which indicated the prognostic value of SNHG1 [33, 34]. They also identified several miRNA-SNHG1 subnetworks (such as hsamiR-195-5p-SNHG1, hsa-miR-497-5p-SNHG1, and hsa-miR-101-3p-SNHG1) and several mRNA-SNHG1 pairs (such as MYLK-AS1-SNHG1, CHEK1-SNHG1, and KIF2C-SNHG1) in the ceRNA regulatory network of HCC $[33,34]$. Through ceRNA network studies, SNHG1 was found to be important in HCC (SNHG1-hsa-miR101-3p-KIF2C) [33], colorectal cancer (SNHG1-hsamiR-484-ORC6, SNHG1-has-miR-423-5p-EZH2, and SNHG1-let-7b-5p-ATP6V1F) [35], lung adenocarcinoma 


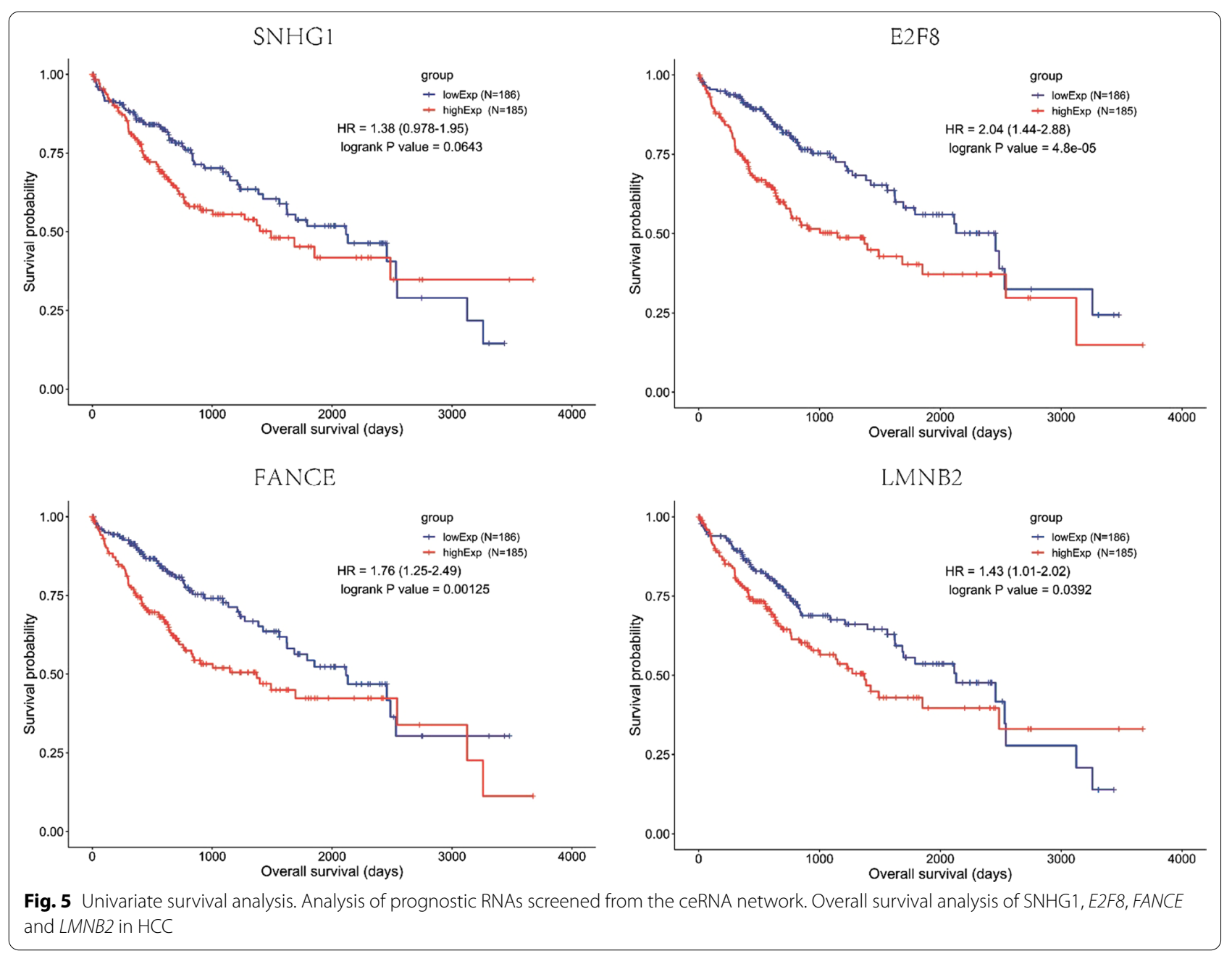

(SNHG1-hsa-miR-21-5p-RALGPS2, SNHG1-hsa-miR326-RALGPS2, SNHG1-hsa-miR-377-3p-RALGPS2) [36], nasopharyngeal carcinoma (SNHG1-miR-145-5pNUAK1) [37] and prostate cancer (SNHG1-miR-199a3p-CDK7) [38]. SNHG1 was also found to be highly expressed in posterior longitudinal ligament patients [39], cholangiocarcinoma [40], and osteosarcoma [41, 42]. In the ceRNA network established in this study, we found SNHG1-hsa-miR-421-E2F8, SNHG1-hsa-miR377-3p-E2F8, SNHG1-hsa-miR-330-5p-LMNB2, and SNHG1-hsa-miR-326-FANCE axes in HCC. E2F8 was found to be required for cell cycle progression and proliferation [43], FANCE to be essential for protection against chromosome breakage [44], and $L M N B 2$ to be required for early replication and transcription [45]. These genes mediate links between miRNAs, which affect the development of HCC as a network. However, studies with large samples are needed to confirm how these miRNAs are connected.
SNHG1 is a lncRNA that is generally thought to be produced by a small nuclear RNA host gene. Cell proliferation and changes in the tumour protein p53 occur when SNHG1 is abnormally expressed [46]. Considering the very important functions of SNHG1, its 3 coexpressed genes can be expected to be related to disease, with altered expression in many human diseases. Of these genes, $E 2 F 8$ is usually accompanied by overexpression of genes during progression of the cell cycle and cell proliferation [47]. Expression of E2F target genes is required in the $\mathrm{S}$ phase of the cell cycle. As a part of this nuclear complex, FANCE interacts with BRCA1 at sites of DNA damage by activating the downstream protein FANCD2 [44]. $L M N B 2$, replicated in the first minute of $S$ phase in humans, is essential for early replication and transcription [48]. In addition, LMNB2, which is located on the inner side of the nuclear envelope, is required for nuclear stability, chromatin structure, and gene expression [48]. 


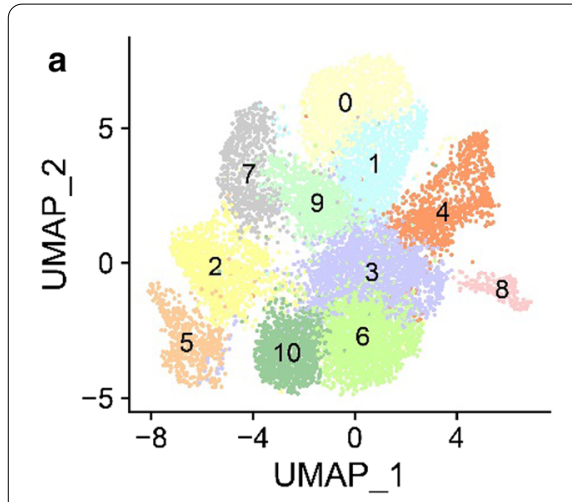

c

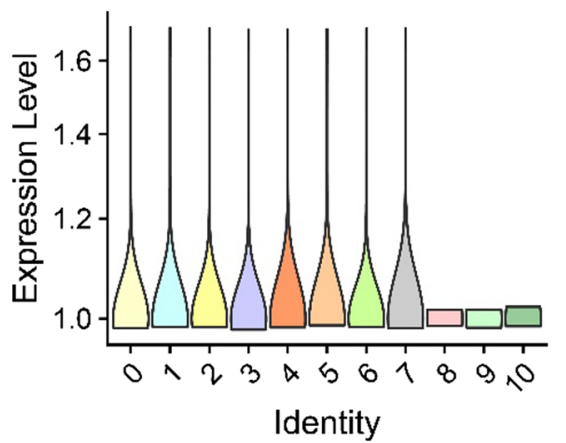

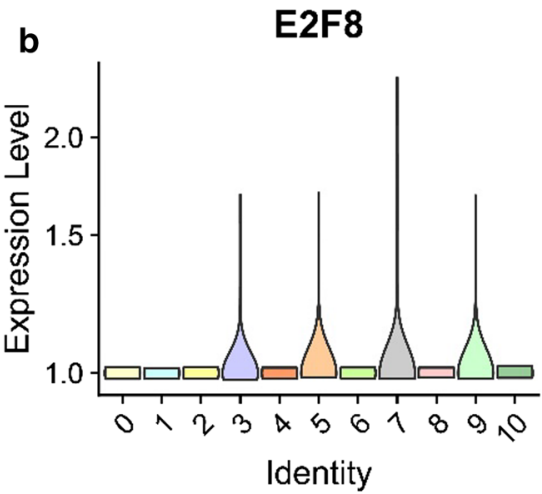

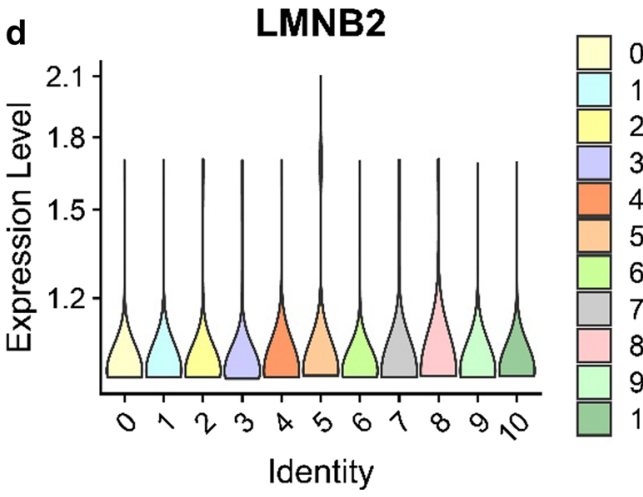

CD9+ ECs Adjacent Normal $1 \mathrm{CD} 9+\mathrm{ECs}$ Adjacent Normal 2 PLVAP+ ECs Peripheral tumor 3 PLPP3+ ECs Core tumor 4 CD320+ ECs Adjacent Normal 5 PLVAP+ ECs Core tumor 6 PLPP3+ ECs Core tumor 7 IGFBP3+ ECs Peripheral tumor 8 TFF3+ ECs Adjacent Normal 9 IGFBP3+ ECs Core tumor 10 PLVAP+ ECs Peripheral tumor

Fig. 6 Tumor-Associated Endothelial Cells. a UMAP analysis and graph-based clustering results were obtained from [26]. Identifies 11 endothelial cell clusters from 14 HCC patients and one healthy donor in liver. b-d Violin graph represent gene expression in endothelial cell population. ECs: endothelial cells
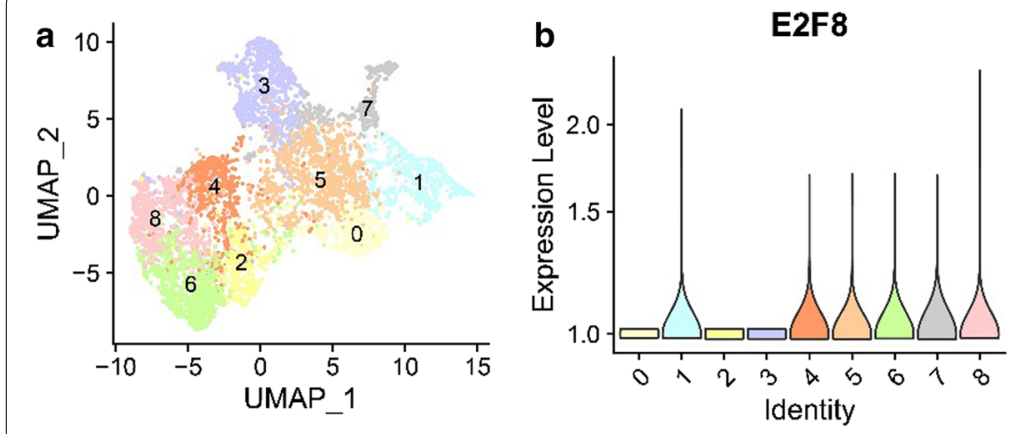

C

FANCE

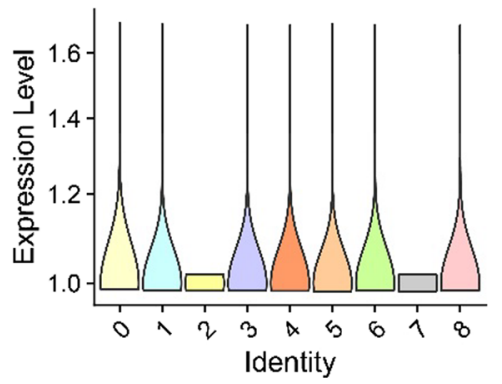

d

LMNB2

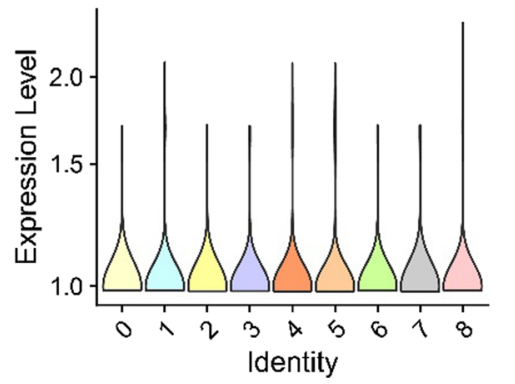

O DC2 Peripheral tumor + Adjacent Normal 1 DC1 Peripheral tumor + Adjacent Normal 2 TAM1 Peripheral tumor + Core tumor 3 Monocyte (Mo) Peripheral tumor + Adjacent Normal 4 TAM3 Peripheral tumor + Core tumor 5 DC1 Peripheral tumor + Adjacent Normal 6 TAM1 Peripheral tumor + Core tumor $7 \mathrm{pDC}$ Peripheral tumor + Adjacent Normal 8 TAM2 Peripheral tumor + Core tumor

Fig. 7 Tumor-Associated Myeloid Cells a UMAP analysis and graph-based clustering results were obtained from [26]. Identifies 9 Myeloid cell clusters from 14 HCC patients and one healthy donor in liver. b-d Violin graph represent gene expression in Myeloid cell population. DC1, DC2, pDC: dendritic cells; TAMs: tumor-associated macrophages 
To explore the cell type specifically of these genes in tumour and normal samples, we analysed their expression in endothelial cells and macrophages and found low expression of $E 2 F 8$ in normal epithelial cells, which may indicate that its presence promotes cancer. This is also consistent with the conclusion drawn from TCGA data, whereby no difference between $L M N B 2$ and FANCE was detected in these two cell types from normal and tumour samples.

\section{Conclusions}

In this work, we identified 7 lncRNA-miRNA-mRNA interactions. Furthermore, we demonstrated that ADAMTSL3, AUTS2, BHLHE40, DUSP6, ERRFI1, GADD45A, GNPNAT1, HIVEP1, MYLK, MYO10, PNRC1, RIPOR2, SMAD6, SPRY2, and SYBU show the same expression trends as SNHG1 in HCC. By generating lncRNA-miRNA-mRNA interaction networks and performing univariate survival analysis of the genes coexpressed with lncRNAs in $\mathrm{HCC}$, we demonstrated that SNHG1 has the same effect on patient survival as $E 2 F 8$, $F A N C E$ and $L M N B 2$. Single-cell analysis also demonstrated that $E 2 F 8$ may play an important role in tumorigenesis or cancer development. Therefore, SNHG1, E2F8, FANCE and LMNB2 may represent therapeutic targets for HCC with complex regulation. Taken together, our research provides genetic resources for capturing the majority of essential genes involved, which will yield a global view of the minimum set of genes and pathways. The biological interaction network of SNHG1-related lncRNA-miRNA-mRNA interactions provides insight into the function of SNHG1 and its molecular regulation in HCC.

\begin{abstract}
Abbreviations
HCC: Hepatocellular carcinoma; GEPIA: Gene Expression Profiling Interactive Analysis; LIHC: Liver hepatocellular carcinoma; SNHG1: Small nucleolar RNA host gene 1; MiRNA: MicroRNA; LncRNA: Long noncoding RNA; ceRNAs: Competing endogenous RNAs; DEGs: Differentially expressed genes; DEMs: Differentially expressed miRNAs; GO: Gene Ontology; PPI: Protein-protein interaction; TCGA: The Cancer Genome Atlas; E2F8: E2F transcription factor 8; FANCE: FA complementation group E; LMNB2: Lamin B2.
\end{abstract}

\section{Acknowledgements}

We thank the editor and reviewers for taking the time and effort to provide insightful and critical discussion.

\section{Authors' contributions}

CZ and SY conceived the study. SY collected raw data and analysed the GDC data. CZ performed the Cytoscape analysis. SY performed the gene function and analysis. CZ and SY wrote the manuscript. All authors read and approved the final manuscript.

\section{Funding}

This study was supported by an unrestricted grant from the Guangdong Postdoctoral Research Foundation (CN) (O0390302). The funders had no role in study design, data collection and analysis, decision to publish, or preparation of the manuscript.
Availability of data and material

The RNA-seq, miRNA and clinical data of LIHC (Liver hepatocellular carcinoma) were downloaded from the DATA PORTAL of GDC (Genomic Data Commons) https://portal.gdc.cancer.gov/projects/TCGA-LIHC. Immunohistochemistry data were downloaded from the Human Protein Atlas database (https:// www.proteinatlas.org/) under gene symbols described in Table 1. Single-cell sequencing data is available from NCBI Gene Expression Omnibus (GEO: GSE156337)

\section{Ethics approval and consent to participate}

Not applicable.

\section{Consent for publication}

Not applicable.

\section{Competing interests}

We declare that we have no conflict of interest.

\section{Author details}

${ }^{1}$ Key Laboratory of Combinatorial Biosynthesis and Drug Discovery, School of Pharmaceutical Sciences, Wuhan University, Wuhan 430071, China. ${ }^{2}$ Guangzhou Institutes of Biomedicine and Health, Chinese Academy of Sciences, 190 Kaiyuan Avenue, Guangzhou Science Park, Luogang District, Guangzhou 510530, China. ${ }^{3}$ Guangzhou Regenerative Medicine and Health Guangdong Laboratory, Bioland Laboratory, Guangzhou 510005, China.

Received: 10 October 2020 Accepted: 17 January 2021

Published online: 26 January 2021

\section{References}

1. Bray F, Ferlay J, Soerjomataram I, et al. Global cancer statistics 2018: GLOBOCAN estimates of incidence and mortality worldwide for 36 cancers in 185 countries. CA Cancer J Clin. 2018;68(6):394-424.

2. Ferlay J, Soerjomataram I, Dikshit R, et al. Cancer incidence and mortality worldwide: sources, methods and major patterns in GLOBOCAN 2012. Int J Cancer. 2015;136(5):E359-86.

3. Chayanupatkul $M, O$ Omino $R$, Mittal $S$, et al. Hepatocellular carcinoma in the absence of cirrhosis in patients with chronic hepatitis B virus infection. J Hepatol. 2017;66(2):355-62.

4. Turati $F$, Edefonti $V$, Talamini R, et al. Family history of liver cancer and hepatocellular carcinoma. Hepatology. 2012;55(5):1416-25.

5. Fu J, Wang H. Precision diagnosis and treatment of liver cancer in China. Cancer Lett. 2018;412:283-8.

6. Anastasiadou E, Jacob LS, Slack FJ. Non-coding RNA networks in cancer. Nat Rev Cancer. 2018;18(1):5-18.

7. Bonasio R, Shiekhattar R. Regulation of transcription by long noncoding RNAs. Annu Rev Genet. 2014;48:433-55.

8. St Laurent G, Wahlestedt C, Kapranov P. The landscape of long noncoding RNA classification. Trends Genet. 2015;31(5):239-51.

9. Luo J, Wang K, Yeh S, et al. LncRNA-p21 alters the antiandrogen enzalutamide-induced prostate cancer neuroendocrine differentiation via modulating the EZH2/STAT3 signaling. Nat Commun. 2019;10(1):2571.

10. Qu L, Wu Z, LiY, et al. A feed-forward loop between IncARSR and YAP activity promotes expansion of renal tumour-initiating cells. Nat Commun. 2016;7:12692.

11. Chowdhury TA, Koceja C, Eisa-Beygi S, et al. Temporal and spatial posttranscriptional regulation of zebrafish tie1 mRNA by long noncoding RNA during brain vascular assembly. Arterioscler Thromb Vasc Biol. 2018;38(7):1562-75.

12. Augoff K, McCue B, Plow EF, et al. miR-31 and its host gene IncRNA LOC554202 are regulated by promoter hypermethylation in triple-negative breast cancer. Mol Cancer. 2012;11:5.

13. Tan $X$, Banerjee $P$, Liu $X$, et al. The epithelial-to-mesenchymal transition activator ZEB1 initiates a prometastatic competing endogenous RNA network. J Clin Invest. 2018;128(7):3198.

14. Braconi C, Kogure T, Valeri N, et al. microRNA-29 can regulate expression of the long non-coding RNA gene MEG3 in hepatocellular cancer. Oncogene. 2011;30(47):4750-6. 
15. Li R, Qu H, Wang S, et al. GDCRNATools: an R/Bioconductor package for integrative analysis of InCRNA, miRNA and mRNA data in GDC. Bioinformatics. 2018;34(14):2515-7.

16. Robinson MD, McCarthy DJ, Smyth GK. edgeR: a Bioconductor package for differential expression analysis of digital gene expression data. Bioinformatics. 2010;26(1):139-40.

17. Ritchie ME, Phipson B, Wu D, et al. limma powers differential expression analyses for RNA-sequencing and microarray studies. Nucleic Acids Res. 2015:43(7):e47.

18. Tang Z, Li C, Kang B, et al. GEPIA: a web server for cancer and normal gene expression profiling and interactive analyses. Nucleic Acids Res. 2017;45(W1):W98-102.

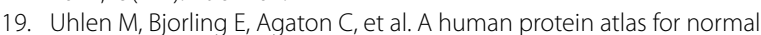
and cancer tissues based on antibody proteomics. Mol Cell Proteomics. 2005:4(12):1920-32

20. Thul PJ, Lindskog C. The human protein atlas: a spatial map of the human proteome. Protein Sci. 2018;27(1):233-44.

21. Zhang $X$, Feng $H$, Li D, et al. Identification of differentially expressed genes induced by aberrant methylation in oral squamous cell carcinomas using integrated bioinformatic analysis. Int J Mol Sci. 2018;19(6):1698.

22. Li JH, Liu S, Zhou H, et al. starBase v2.0: decoding miRNA-ceRNA, miRNAncRNA and protein-RNA interaction networks from large-scale CLIP-Seq data. Nucleic Acids Res. 2014;42(Database issue):92-7.

23. Otasek D, Morris JH, Boucas J, et al. Cytoscape Automation: empowering workflow-based network analysis. Genome Biol. 2019;20(1):185.

24. Shannon P, Markiel A, Ozier O, et al. Cytoscape: a software environment for integrated models of biomolecular interaction networks. Genome Res. 2003;13(11):2498-504.

25. Zhou Y, Zhou B, Pache L, et al. Metascape provides a biologist-oriented resource for the analysis of systems-level datasets. Nat Commun. 2019:10(1):1523.

26. Sharma A, Seow JJW, Dutertre CA, et al. Onco-fetal reprogramming of endothelial cells drives immunosuppressive macrophages in hepatocellular carcinoma. Cell. 2020;183:377-94.

27. Stuart T, Butler A, Hoffman P, et al. Comprehensive integration of singlecell data. Cell. 2019;177(7):1888-1902 e21.

28. Sahu D, Hsu CL, Lin CC, et al. Co-expression analysis identifies long noncoding RNA SNHG1 as a novel predictor for event-free survival in neuroblastoma. Oncotarget. 2016;7(36):58022-37.

29. Li J, Ran C, Li E, et al. Synergistic function of E2F7 and E2F8 is essential for cell survival and embryonic development. Dev Cell. 2008;14(1):62-75.

30. Bouffard F, Plourde $K$, Belanger $S$, et al. Analysis of a FANCE splice isoform in regard to DNA repair. J Mol Biol. 2015;427(19):3056-73.

31. Misono S, Seki N, Mizuno K, et al. Dual strands of the miR-145 duplex (miR-145-5p and miR-145-3p) regulate oncogenes in lung adenocarcinoma pathogenesis. J Hum Genet. 2018;63(10):1015-28.

32. Liu XH, Sun M, Nie FQ, et al. Lnc RNA HOTAIR functions as a competing endogenous RNA to regulate HER2 expression by sponging miR-331-3p in gastric cancer. Mol Cancer. 2014;13:92.

33. Zhang J, Lou W. A key mRNA-miRNA-IncRNA competing endogenous RNA triple sub-network linked to diagnosis and prognosis of hepatocellular carcinoma. Front Oncol. 2020;10:340.
34. Lin P, Wen DY, Li Q, et al. Genome-wide analysis of prognostic IncRNAs, miRNAs, and mRNAs forming a competing endogenous RNA network in hepatocellular carcinoma. Cell Physiol Biochem. 2018;48(5):1953-67.

35. Qi X, Lin Y, Liu X, et al. Biomarker discovery for the carcinogenic heterogeneity between colon and rectal cancers based on IncRNA-associated ceRNA network analysis. Front Oncol. 2020;10:535985.

36. Tan J, Wang W, Song B, et al. Integrative analysis of three novel competing endogenous RNA biomarkers with a prognostic value in lung adenocarcinoma. Biomed Res Int. 2020;2020:2837906.

37. Lan X, Liu X. LncRNA SNHG1 functions as a ceRNA to antagonize the effect of miR-145a-5p on the down-regulation of NUAK1 in nasopharyngeal carcinoma cell. J Cell Mol Med. 2019;23(4):2351-61.

38. Li J, Zhang Z, Xiong L, et al. SNHG1 IncRNA negatively regulates miR199a-3p to enhance CDK7 expression and promote cell proliferation in prostate cancer. Biochem Biophys Res Commun. 2017;487(1):146-52.

39. Wang $Y$, Niu H, Liu Y, et al. Promoting effect of long non-coding RNA SNHG1 on osteogenic differentiation of fibroblastic cells from the posterior longitudinal ligament by the microRNA-320b/IFNGR1 network. Cell Cycle. 2020;19(21):2836-50.

40. Li Z, Li X, Du X, et al. The interaction between IncRNA SNHG1 and miR140 in regulating growth and tumorigenesis via the TLR4/NF-kappaB pathway in cholangiocarcinoma. Oncol Res. 2019;27(6):663-72.

41. Wang J, Cao L, Wu J, et al. Long non-coding RNA SNHG1 regulates NOB1 expression by sponging miR-326 and promotes tumorigenesis in osteosarcoma. Int J Oncol. 2018;52(1):77-88.

42. Jiang Z, Jiang C, Fang J. Up-regulated InC-SNHG1 contributes to osteosarcoma progression through sequestration of miR-577 and activation of WNT2B/Wnt/beta-catenin pathway. Biochem Biophys Res Commun. 2018:495(1):238-45

43. Maiti B, Li J, de Bruin A, et al. Cloning and characterization of mouse E2F8, a novel mammalian E2F family member capable of blocking cellular proliferation. J Biol Chem. 2005;280(18):18211-20.

44. Pace $P$, Johnson M, Tan WM, et al. FANCE: the link between Fanconi anaemia complex assembly and activity. EMBO J. 2002;21(13):3414-23.

45. Giacca M, Zentilin L, Norio P, et al. Fine mapping of a replication origin of human DNA. Proc Natl Acad Sci USA. 1994;91(15):7119-23.

46. Shen Y, Liu S, Fan J, et al. Nuclear retention of the IncRNA SNHG1 by doxorubicin attenuates hnRNPC-p53 protein interactions. EMBO Rep. 2017;18(4):536-48.

47. Christensen J, Cloos P, Toftegaard U, et al. Characterization of E2F8, a novel E2F-like cell-cycle regulated repressor of E2F-activated transcription. Nucleic Acids Res. 2005;33(17):5458-70.

48. Biamonti G, Giacca M, Perini G, et al. The gene for a novel human lamin maps at a highly transcribed locus of chromosome 19 which replicates at the onset of S-phase. Mol Cell Biol. 1992;12(8):3499-506.

\section{Publisher's Note}

Springer Nature remains neutral with regard to jurisdictional claims in published maps and institutional affiliations.

Ready to submit your research? Choose BMC and benefit from

- fast, convenient online submission

- thorough peer review by experienced researchers in your field

- rapid publication on acceptance

- support for research data, including large and complex data types

- gold Open Access which fosters wider collaboration and increased citations

- maximum visibility for your research: over 100M website views per year

At BMC, research is always in progress.

Learn more biomedcentral.com/submissions 